\title{
SUMMATION OF THE COEFFICIENTS OF SOME TERMINATING HYPERGEOMETRIC SERIES
}

By W. F. SHeppard.

[Received November 3rd, 1910.-Read November 10th, 1910.-Revised June 28th, 1911.]

1. We adopt the notation*

$$
\begin{array}{ll}
a^{[r]} \equiv \alpha(\alpha+1) \ldots(\alpha+r-1), & a^{(r)} \equiv \alpha(\alpha-1) \ldots(\alpha-r+1), \\
\alpha_{[r]} \equiv \alpha^{[r]} / r !, & a_{(r)} \equiv a^{(r)} / r !,
\end{array}
$$

so that

$$
\begin{aligned}
& a^{[r]}=(\alpha+r-1)^{(r)}, \quad a^{(r)}=(\alpha-r+1)^{[r]} \\
& (-\alpha)^{[r]}=(-)^{r} \alpha^{(r)}=(-)^{r}(\alpha-r+1)^{[r]} \text {, } \\
& (-\alpha)^{(r)}=(-)^{r} \alpha^{[r]}=(-)^{r}(a+r-1)^{(r)} \\
& \alpha^{[r+8]}=\alpha^{[r]}(\alpha+\gamma)^{[s]}, \\
& a^{(r+s)}=a^{(r)}(\alpha-r)^{(s)} \\
& a^{[r]} / a^{[s]}=(\alpha+s)^{[r-s]}, \quad \quad a^{(r)} / a^{(s)}=(a-s)^{(r-s)}, \\
& (\alpha+r)^{[s]} / \alpha^{[s]}=(a+s)^{[r]} / \alpha^{[r]}, \quad(a-r)^{(s)} / \alpha^{(s)}=(\alpha-s)^{(r)} / \alpha^{(r)}
\end{aligned}
$$

Also we write

$$
F\{a, \beta ; \rho, \theta ; x\} \equiv 1+\frac{\alpha \cdot \beta}{\rho \cdot \theta} x+\frac{\alpha(\alpha+1) \cdot \beta(\beta+1)}{\rho(\rho+1) \cdot \theta(\theta+1)} x^{2}+\ldots,
$$

$F\{\alpha, \beta, \gamma ; \rho, \theta, \phi ; x\} \equiv 1+\frac{\alpha \cdot \beta \cdot \gamma}{\rho \cdot \theta \cdot \phi} x+\frac{\alpha(\alpha+1) \cdot \beta(\beta+1) \cdot \gamma(\gamma+1)}{\rho(\rho+1) \cdot \theta(\theta+1) \cdot \phi(\phi+1)} x^{2}+\ldots$,

and we denote the sum of the first $r+1$ terms of the series $F\{\ldots\}$ by $F_{r}\{\ldots\}$.

The general term of the generalised series, taking $x=1$, is

$$
y_{r} \equiv \frac{\alpha^{[r]} \beta^{[r]} \gamma^{[r]} \ldots(m \text { factorials })}{\rho^{[r]} \theta^{[r]} \phi^{[r]} \ldots(m \text { factorials })} .
$$

The properties of any particular series depend to a considerable extent on the value of

$$
D \equiv\{\rho+\theta+\phi+\ldots\}-\{a+\beta+\gamma+\ldots\} .
$$


The value of $D$ can usually be more conveniently obtained from the general term, written in the form

$$
y_{r}=\frac{[\ldots(\alpha+r-1)][\ldots(\beta+r-1)][\ldots(\gamma+r-1)] \ldots}{[\ldots(\rho+r-1)][\ldots(\theta+r-1)][\ldots(\phi+r-1)] \ldots},
$$

where $[\ldots(a+r-1)]$ denotes an ascending factorial ending with $a+r-1$; for the addition of factors at the beginning of one of the factorials in each term does not alter the mutual ratios of the terms, but only multiplies or divides the series as a whole by these factors. We have then

$$
\begin{aligned}
D=\{(\rho+r-1)+ & (\theta+r-1)+(\phi+r-1)+\ldots\} \\
& -\{(\alpha+r-1)+(\beta+r-1)+(\gamma+r-1)+\ldots\} .
\end{aligned}
$$

We are only concerned with the cases in which $a=-n$, where $n$ is a positive integer, so that the series terminates with the $(n+1)$ th term; it being assumed that the denominator is not zero in any of the first $n+1$ terms.

If, in addition, $\rho=1$, so that $\rho^{[r]}=r !$, then $F\{\alpha, \beta ; \rho, \theta ; x\}$ becomes

$$
F\{-n, \beta ; 1, \theta ; x\}=1-n_{(1)} \frac{\beta}{\theta} x+n_{(2)} \frac{\beta(\beta+1)}{\theta(\theta+1)} x^{2}-\ldots,
$$

and the sum of the coefficients in the complete series of $n+1$ terms is

and similarly

$$
F_{n}\{-n, \beta ; 1, \theta ; 1\}=\sum_{r=0}^{r=n}(-)^{r} n_{(r)} \beta^{[r]} / \theta^{[r]} ;
$$

$$
F_{n}\{-n, \beta, \gamma ; 1, \theta, \phi ; 1\}=\sum_{r=0}^{r=n}(-)^{r} n_{(r)}\left\{\beta^{[r]} \gamma^{[r]}\right\} /\left\{\theta^{[r]} \phi^{[r]}\right\},
$$

$F_{n}\{-n, \beta, \gamma, \delta ; 1, \theta, \phi, \psi ; 1\}=\sum_{r=0}^{r=n}(-)^{r} n_{(r)}\left\{\beta^{[r]} \gamma^{[r]} \delta^{[r]}\right\} /\left\{\theta^{[r]} \phi^{[r]} \psi^{[r]}\right\}$.

For this class of cases we have

$$
D-2=\{\theta+\phi+\psi+\ldots\}-\{\beta+\gamma+\delta+\ldots\}+n-1 .
$$

\section{Four-Factorial Series.}

2. For the ordinary series we know that

$$
F_{n}\{\rightarrow n, \beta ; 1, \theta ; 1\}=(\theta-\beta)^{[n]} / \theta^{[n]} .
$$

We can prove this in either of two ways.

(i) The simplest method, for this particular class of cases, is by induction for $n=1,2,3, \ldots$. Suppose that (2) is true for a particular value 
of $n$. Then

$$
\begin{aligned}
F_{n+1}\{-n-1, & \beta ; 1, \theta ; 1\} \\
& =\sum_{r=0}^{r=n+1}(-)^{r}(n+1)_{(r)} \beta^{[r]} / \theta^{[r]} \\
& =1+\sum_{r=1}^{r=n}(-)^{r}\left\{n_{(r)}+n_{(r-1)}\right\} \beta^{[r]} / \theta^{[r]}+(-)^{n+1} \beta^{[n+1]} / \theta^{[n+1]} \\
& =\sum_{r=0}^{r=n}(-)^{r} n_{(r)} \beta^{[r]} / \theta^{[r]}-(\beta / \theta) \sum_{r=0}^{r=n}(-)^{r} n_{(r)}(\beta+1)^{[r]} /(\theta+1)^{[r]} \\
& =(\theta-\beta)^{[n]} / \theta^{[n]}-(\beta / \theta)(\theta-\beta)^{[n]} /(\theta+1)^{[n]} \\
& =\{(\theta+n)-\beta\}(\theta-\beta)^{[n]} / \theta^{[n+1]} \\
& =(\theta-\beta)^{[n+1]} / \theta^{[n+1]} .
\end{aligned}
$$

But

$$
F_{1}\{-1, \beta ; 1, \theta ; 1\}=1-\beta / \theta=(\theta-\beta) / \theta ;
$$

hence the induction holds.

(ii) An alternative method has been given by M. J. M. Hill.* $\mathrm{He}$ shows by induction for $s=1,2,3, \ldots$, that

$$
\begin{gathered}
F_{s}\{\alpha, \beta ; 1, \theta ; 1\}-\frac{\theta-\alpha-\beta-1}{\theta} F_{s-1}\{\alpha+1, \beta+1 ; 1, \theta+1 ; 1\} \\
=\frac{(\alpha+1)^{[s]}(\beta+1)^{[s]}}{1^{[s]} \theta^{[s]}} ;
\end{gathered}
$$

and therefore, putting $a=-n, s=n$, $F_{n}\{-n, \beta ; 1, \theta ; 1\}=\frac{\theta-\beta+n-1}{\theta} F_{n-1}\{-(n-1), \beta+1 ; 1, \theta+1 ; 1\}$. (4) Replacing $n$ by $n-1, n-2, \ldots, 1$, and aggregating the results, we obtain (2).

For the case of $D=2$, so that $\theta-\alpha-\beta-1=0$, (3) gives

$$
F_{s}\{\alpha, \beta ; 1, \theta ; 1\}=\left\{(\alpha+1)^{[s]}(\beta+1)^{[s]}\right\} /\left\{s ! \theta^{[s]}\right\} .
$$

3. It follows from (2) that $F_{n}\{-n, \beta ; 1, \theta ; 1\}$ is zero if any one of the factors of $(\theta-\beta)^{[n]}$ is zero. Suppose, therefore, that

$$
\theta-\beta+r=0 \text {, }
$$

giving

$$
D-2=(n-1)-r \text {, }
$$


where $r$ has any of the values $0,1,2, \ldots, n-1$. Then

$$
\begin{aligned}
0=F_{n}\{-n, \theta+r ; 1, \theta ; 1\} & =\sum_{s=0}^{s=n}(-)^{s} n_{(s)}(\theta+r)^{[s]} / \theta^{[s]} \\
& =\sum_{s=0}^{s=n}(-)^{s} n_{(s)}(\theta+s)^{[r]} / \theta^{[r]},
\end{aligned}
$$

by (1); and therefore

$$
\sum_{s=0}^{s=n}(-)^{s} n_{(s)}(\theta+s)^{[r]}=0
$$

But, if $f_{r}(\theta)$ is any polynomial of degree $r$ in $\theta$, we can express $f_{r}(\theta)$ in the form

$$
f_{r}(\theta)=a_{r} \theta^{[r]}+a_{r-1} \theta^{[r-1]}+\ldots+a_{1} \theta+a_{0} ;
$$

and therefore, replacing $r$ in (5) by $r-1, r-2, \ldots, 0$, it follows that

$$
\sum_{s=0}^{s=n}(-)^{s} n_{(s)} f_{r}(\theta+s)=0 \quad(r=0,1,2, \ldots, n-1) .
$$

This is only another way of stating the more familiar theorem that

$$
\sum_{s=0}^{s=n}(-)^{s} n_{(s)} s^{r}=0 \quad(r=0,1,2, \ldots, n-1) .
$$

4. Now let us see whether we can sum the more general fourfactorial series

$$
F\{a, \beta ; \rho, \theta ; 1\} \equiv 1+\frac{a . \beta}{\rho . \theta}+\ldots+\frac{a^{[s]} \beta^{[s]}}{\rho^{[s]} \theta^{[s]}}+\ldots,
$$

for the case of $a=-n$. Adapting Hill's method, quoted above, let us write

$$
\begin{aligned}
& f(s) \equiv F_{s}\{\alpha, \beta ; \rho, \theta ; 1\} \\
& -\frac{\left(\rho+p \alpha+p^{\prime} \beta+q\right)\left(\theta+p \beta+p^{\prime} \alpha+q\right)}{\rho \theta} F_{s-1}\{\alpha+1, \beta+1 ; \rho+1, \theta+1 ; 1\},
\end{aligned}
$$

where $p, p^{\prime}$, and $q$ are constants whose values are to be chosen. Then

$$
\rho \theta . f(1)=\rho \theta+\alpha \beta-\left(\rho+p \alpha+p^{\prime} \beta+q\right)\left(\theta+p \beta+p^{\prime} \alpha+q\right) .
$$

Replacing $\rho$ by $D+a+\beta-\theta$, we find that, in order that $f(1)$ may contain $\alpha+1$ and $\beta+1$ as factors, we must have

$$
p^{\prime}=p, \quad q=-1, \quad D=2,
$$

and either

$$
p=0 \text { or } p=-1 \text {; }
$$


but the two cases are really identical, since $D=2$ gives

$$
\rho-\alpha-\beta-1=-(\theta-1), \quad \theta-\alpha-\beta-1=-(\rho-1) .
$$

Taking $p=0$, we can show by induction that, for this value of $D$,

$$
\begin{gathered}
F_{s}\{\alpha, \beta ; \rho, \theta ; 1\}-\frac{(\rho-1)(\theta-1)}{\rho \theta} F_{s-1}\{\alpha+1, \beta+1 ; \rho+1, \theta+1 ; 1\} \\
=\frac{(a+1)^{[s]}(\beta+1)^{[s]}}{\rho^{[s]} \theta^{[s]}}(\rho+\theta=\alpha+\beta+2) .
\end{gathered}
$$

This is true whatever the value of $a$ may be. Writing $a=-n, s=n$, and replacing $\beta$ by $\rho+\theta-\alpha-2$, we obtain

$$
\begin{aligned}
& F_{n}\{-n, \rho+\theta+n-2 ; \rho, \theta ; 1\} \\
& \quad=\frac{(\rho-1)(\theta-1)}{\rho \theta} F_{n-1}\{-n+1, \rho+\theta+n-1 ; \rho+1, \theta+1 ; 1\} .(9)
\end{aligned}
$$

Replacing $n$ by $n-1, n-2, \ldots, 1$, and aggregating the results, we have, finally,

$$
\begin{aligned}
F_{n}\{-n, \rho+\theta+n-2 ; \rho, \theta ; 1\} & =\frac{(\rho-1)^{[n]}(\theta-1)^{[n]}}{\rho^{[n]} \theta^{[n]}} \\
& =\frac{(\rho-1)(\theta-1)}{(\rho+n-1)(\theta+n-1)} .
\end{aligned}
$$

Six-Factorial Series.

5. Next consider the six-factorial series

$$
F\{\alpha, \beta, \gamma ; 1, \theta, \phi ; 1\} \equiv 1+\frac{\alpha \cdot \beta \cdot \gamma}{1 \cdot \theta \cdot \phi}+\frac{a(\alpha+1) \cdot \beta(\beta+1) \cdot \gamma(\gamma+1)}{2 ! \theta(\theta+1) \cdot \phi(\phi+1)}+\ldots,
$$

of which the series considered in $\$ 4$ is a particular case. It will be found, as in $\S 4$, that Hill's method can be applied if $D=2$, i.e., if

We have

$$
\gamma=\theta+\phi-\alpha-\beta-1 \text {. }
$$

$$
\begin{aligned}
1+\frac{\alpha \beta(\theta+\phi-\alpha-\beta-1)}{\theta \phi}-\frac{(\theta-\alpha-\beta-1)(\phi-\alpha-\beta-1)}{\theta \phi} & \\
= & \frac{(\alpha+1)(\beta+1)(\theta+\phi-\alpha-\beta-1)}{\theta \phi} ;
\end{aligned}
$$


and thence, by induction for $s=1,2,3, \ldots$, we can show that

$$
\begin{aligned}
F_{s}\{a, \beta, \theta+\phi- & a-\beta-1 ; 1, \theta, \phi ; 1\}-\frac{(\theta-a-\beta-1)(\phi-a-\beta-1)}{\theta \phi} \\
& \times F_{s-1}\{\alpha+1, \beta+1, \theta+\phi-a-\beta-1 ; 1, \theta+1, \phi+1 ; 1\} \\
& =\frac{(\alpha+1)^{[s]}(\beta+1)^{[s]}(\theta+\phi-a-\beta-1)^{[s]}}{s ! \theta^{[s]} \phi^{[s]}}
\end{aligned}
$$

This is true whatever the value of $a$ may be. Putting $\alpha=-n, s=n$, we obtain

$$
\begin{aligned}
& F_{n}\{-n, \beta, \theta+\phi-\beta+n-1 ; 1, \theta, \phi ; 1\} \\
& =\frac{\theta-\beta+n-1}{\theta} \frac{\phi-\beta+n-1}{\phi} \\
& \quad \times F_{n-1}\{-n+1, \beta+1, \theta+\phi-\beta+n-1 ; 1, \theta+1, \phi+1 ; 1\} .
\end{aligned}
$$

Replacing $n$ by $n-1, n-2, \ldots, 1$, and aggregating the results, we have

$$
\begin{aligned}
F_{n}\{-n, \beta, \theta+\phi-\beta+n-1 ; 1, \theta, \phi ; 1\} \\
=\frac{(\theta-\beta+n-1)^{(n)}}{\theta^{[n]}} \frac{(\phi-\beta+n-1)^{(n}}{\phi^{[n]}}=\frac{(\theta-\beta)^{[n]}(\phi-\beta)^{[n]}}{\theta^{[n]} \phi^{[n]}} .
\end{aligned}
$$

We ought also to have, by symmetry,

$$
F_{n}\{-n, \beta, \theta+\phi-\beta+n-1 ; 1, \theta, \phi ; 1\}=\frac{(\theta-\gamma)^{[n]}(\phi-\gamma)^{[n]}}{\theta^{[n]} \phi^{[n]}},
$$

where $\gamma \equiv \theta+\phi-\beta+n-1$. This is easily verified ; for

$$
\begin{gathered}
\theta-\gamma=-(\phi-\beta+n-1), \quad \phi-\gamma=-(\theta-\beta+n-1), \\
(\theta-\gamma)^{[n]}=(-)^{n}(\phi-\beta+n-1)^{(n)}=(-)^{n}(\phi-\beta)^{[n]}, \\
(\phi-\gamma)^{[n]}=(-)^{n}(\theta-\beta+n-1)^{(n)}=(-)^{n}(\theta-\beta)^{[n]} .
\end{gathered}
$$

6. By putting $\beta=1$ in (13), we obtain (10). The following are two other special cases.

(i) Let $\phi=\theta$. Then

$$
\begin{aligned}
F_{n}\{-n, \beta, 2 \theta-\beta+n-1 ; 1, \theta, \theta ; 1\} & =\left\{\frac{(\theta-\beta)^{[n]}}{\theta^{[n]}}\right\}^{2} \\
& =\left[F_{n}\{-n, \beta ; 1, \theta ; 1\}\right]^{2} .
\end{aligned}
$$

(ii) Let one of the two numbers $\beta$ and $\gamma$ in the numerator of the second term be equal to the difference of the two $\theta$ and $\phi$ in the 
denominator ; e.g., let $\theta+\phi-\beta+n-1=\phi-\theta$, so that $\beta=2 \theta+n-1$. Then

$$
\begin{aligned}
F_{n}\{-n, 2 \theta+ & n-1, \phi-\theta ; 1, \theta, \phi ; 1\} \\
& =\frac{2 \theta-\phi}{\phi} F_{n-1}\{-n+1,2 \theta+n, \phi-\theta ; 1, \theta+1, \phi+1 ; 1\} \\
& =\ldots \\
& =\frac{(2 \theta-\phi)^{[n]}}{\phi^{[n]}} .
\end{aligned}
$$

7. Having obtained an expression for the sum of the coefficients in the six-factorial series for the case of $D=2$, we can obtain an expression for their sum in the case where $D$ is any positive integer greater than 2. For, if $D=2+k$, where $k$ is a positive integer, and if $y_{s}$ is the $(s-1)$ th term of the series (for $x=1$ ), we can replace $y_{s}$ by $y_{s} \Psi(s)$, where $\Psi(s)$ is the sum of $k+1$ terms, each consisting of $k$ factors so chosen as to extend the factorials in the numerator of $y_{s}$ or reduce the factorials in the denominator, the coefficients of the terms being chosen so as to make $\Psi(s)=1$; and we shall thus replace the series by the sum of $k+1$ series, for each of which $D=2$. This can usually be done in several different ways; but there will be three typical forms, obtained by altering (i) two factorials in the numerator, (ii) two factorials in the denominator, and (iii) one factorial in the numerator and one in the denominator, respectively.

Suppose, for instance, that $D=3$. Then we can use any one of the three relations

$$
\begin{aligned}
1 & =\{(\beta+s)-(\gamma+s)\} /(\beta-\gamma) \\
& =\{(\theta+s-1)-(\phi+s-1)\} /(\theta-\phi) \\
& =\{(\beta+s)-(\phi+s-1)\} /(\beta-\phi+1) .
\end{aligned}
$$

If we use the first, we obtain

$$
\frac{\beta^{[s]} \gamma^{[s]}}{\theta^{[s]} \phi^{[s]}}=\frac{\beta}{\beta-\gamma} \frac{(\beta+1)^{[s]} \gamma^{[s]}}{\theta^{[s]} \phi^{[s]}}-\frac{\gamma}{\beta-\gamma} \frac{\beta^{[s]}(\gamma+1)^{[s]}}{\theta^{[s]} \phi^{[s]}} ;
$$

this gives the general formula.

$$
\begin{aligned}
& (\beta-\gamma) F_{s}\{\alpha, \beta, \gamma ; 1, \theta, \phi ; 1\} \\
& \left.=\beta F_{s} ; \alpha, \beta+1, \gamma ; 1, \theta, \phi ; 1\right\}-\gamma F_{s}\{a, \beta, \gamma+1 ; 1, \theta, \phi ; 1\} ;
\end{aligned}
$$


and thence it will be found that

$$
\begin{aligned}
F_{n}\{-n, \beta, \theta & +\phi-\beta+n-2 ; 1, \theta, \phi ; 1\} \\
& =\frac{(\theta-\beta)^{[n]}(\phi-\beta)^{[n]}+n \beta(\theta-\beta)^{[n-1]}(\phi-\beta)^{[n-1]}}{\theta^{[n]} \phi^{[n]}} \\
& =\frac{(\theta-\beta)^{[n]}(\phi-\beta)^{[n]}}{\theta^{[n]} \phi^{[n]}}\left\{1+\frac{n \beta}{(\theta-\beta+n-1)(\phi-\beta+n-1)}\right\} .
\end{aligned}
$$

Similarly for $D=4$ it may be shown that

$$
\begin{aligned}
& F_{n}\{-n, \beta, \theta+\phi-\beta+n-3 ; 1, \theta, \phi ; 1\} \\
& =\frac{(\theta-\beta)^{[n]}(\phi-\beta)^{[n]}}{\theta^{[n]} \phi^{[n]}}\left\{1+\frac{2 n \beta}{(\theta-\beta+n-1)(\phi-\beta+n-1)}\right. \\
& \left.\quad+\frac{n(n-1) \beta(\beta+1)}{(\theta-\beta+n-1)(\theta-\beta+n-2)(\phi-\beta+n-1)(\phi-\beta+n-2)}\right\} .
\end{aligned}
$$

8. These last results suggest that the formula for the case of $D=2+k$, where $k$ is a positive integer, is

$$
\begin{aligned}
& F_{n}\{-n, \beta, \theta+\phi-\beta+n-k-1 ; 1, \theta, \phi ; 1\} \\
& =\frac{(\theta-\beta)^{[n]}(\phi-\beta)^{[n]}}{\theta^{[n]} \phi^{[n]}} F_{k}\{-n, \beta,-k ; 1,-\theta+\beta-n+1,-\phi+\beta-n+1 ; 1\} .
\end{aligned}
$$

This, however, is only a particular case of a more general formula ; for we can replaee $F_{k}\{\ldots\}$ on the right-hand side by $F_{n}\{\ldots\}$, and we then have a formula which, if it is true for all positive integral values of $k$, must be true universally. Replacing $\theta+\phi-\beta+n-k-1$ by $\gamma$, the formula becomes

$$
\begin{aligned}
& \theta^{[n]} \phi^{[n]} F_{n}\{-n, \beta, \gamma ; 1, \theta, \phi ; 1\} \\
& =(\theta-\beta)^{[n]}(\phi-\beta)^{[n]} F_{n}\{-n, \beta, \beta+\gamma-\theta-\phi-n+1 ; \\
& =(-\theta+\beta-n+1)^{[n]}(-\phi+\beta-n+1)^{[n]} F_{n}\{-n, \beta, \beta+\gamma-\theta-\phi-n+1 ; \\
& \quad 1,-\theta+\beta-n+1,-\phi+\beta-n+1 ; 1\} \quad(18 a) \\
& =\theta^{[n]} \phi^{\prime[n]} F_{n}\left\{-n, \beta, \gamma^{\prime} ; 1, \theta^{\prime}, \phi^{\prime} ; 1\right\},
\end{aligned}
$$

where $\quad \gamma^{\prime}=\beta+\gamma-\theta-\phi-n+1, \quad \gamma=\beta+\gamma^{\prime}-\theta^{\prime}-\phi^{\prime}-n+1$

$$
\begin{array}{ll}
\theta^{\prime}=-\phi+\beta-n+1, & \theta=-\phi^{\prime}+\beta-n+1 \\
\phi^{\prime}=-\theta+\beta-n+1, & \phi=-\theta^{\prime}+\beta-n+1
\end{array}
$$


To zstablish this, let us write

$$
\begin{aligned}
\Phi(n, \beta, \gamma, \theta, \phi) & \equiv \theta^{[n]} \phi^{[n]} F_{n}\{-n, \beta, \gamma ; 1, \theta, \phi ; 1\} \\
& =\sum_{r=0}^{r=n}(-)^{r} n_{(r)} \beta^{[r]} \gamma^{[r]}(\theta+r)^{[n-r]}(\phi+r)^{[n-r]},
\end{aligned}
$$

$$
\begin{gathered}
\Psi(n, \beta, \gamma, \theta, \phi) \equiv(\theta-\beta)^{[n]}(\phi-\beta)^{[n]} F_{n}\{-n, \beta, \beta+\gamma-\theta-\phi-n+1 ; \\
1,-\theta+\beta-n+1,-\phi+\beta-n+1 ; 1\} \\
=\sum_{r=0}^{r=n}()^{r} n_{(r)} \beta^{[r]}(\beta+\gamma-\theta-\phi-n+1)^{[r]} \\
\quad \times(\theta-\beta)^{[n-r]}(\phi-\beta)^{[n-r]}, \\
f_{n}(\gamma) \equiv \Phi(n, \beta, \gamma, \theta, \phi)-\Psi(n, \beta, \gamma, \theta, \phi) ;
\end{gathered}
$$

then we wish to show that, for any assigned values of $\beta, \theta$, and $\phi$, and for any assigned (positive.integral) value of $n, f_{n}(\gamma)$ is 0 for all values of $\gamma$. Suppose this is true for values of $n$ up to $n-1$ inclusive. Then

$$
\begin{aligned}
\Phi(n, \beta, \gamma+1, \theta, \phi)-\Phi(n, \beta, \gamma, \theta, \phi) & =-n \beta \Phi(n-1, \beta+1, \gamma+1, \theta+1, \phi+1), \\
& =-\beta(n, \beta, \gamma+1, \theta, \phi)-\Psi(n, \beta, \gamma, \theta, \phi) \\
& =-n \beta \Psi(n-1, \beta+1, \gamma+1, \theta+1, \phi+1) ;
\end{aligned}
$$

and therefore

$$
f_{n}(\gamma)=f_{n}(\gamma+1)=f_{n}(\gamma+2)=\ldots .
$$

\#lence $f_{n}(\gamma)$ is independent of $\gamma$. Now write

$$
\beta+\gamma-\theta-\phi-x+1=0,
$$

sothat $\Psi$ seduces to its first term; then

$$
\gamma=\theta+\phi-\beta+n-1,
$$

and we see from (13) that $\Phi$ and $\Psi$ are equal, so that $f_{n}(\gamma)=0$. Hence the step of the induction holds. But $f_{n}(\gamma)=0$ for $n=0$. Hence the theorem is true universally.

It will be noticed that, if $D$ and $D^{\prime}$ are the values of $D$ for the two series,

$$
D=2-\gamma^{\prime}, \quad D^{\prime}=2-\gamma,
$$

so that the formula gives us an approximate value for the sum of the coefficients of a terminating six-factorial series for which $D$ differs only slightly from 2.

9. The method of $\S 7$ can be used to obtain an expression for

$$
\sum_{s=0}^{s=n} y_{s} \chi(s)
$$


where $y_{s} x^{s}$ is the $(s+1)$ th term of 8 terminating series for which $D=2+k, k$ being a positive integer, and $\chi(s)$ is a polynomial in $s$ of degree not exceeding $k$; the only difference being that the coefficients of the terms constituting $\Psi(s)$ are to be chosen so as to make $\Psi(s)=\chi(s)$.

Suppose, for instance, that we require the sum of the series

$$
F_{n}\{-n, \beta, \gamma, \epsilon ; 1, \theta, \phi, \psi ; 1\} ;
$$

the constants being subject to the conditions

$$
\epsilon=\psi+k, \quad(1+\theta+\phi+\psi)-(-n+\beta+\gamma+\epsilon)=2,
$$

where $k$ is a positive integer. This is ostensibly an eight-factorial series ; but, since

$$
(\psi+k)^{[s]} / \psi^{[s]}=(\psi+s)^{[k]} / \psi^{i k j},
$$

it is really of the form mentioned above. It will be found that the three typical formulæ are

$$
\begin{aligned}
& F_{n}\{-n, \beta, \gamma, \psi+k ; 1, \theta, \phi, \psi ; 1\} \\
& =\sum_{t=0}^{t=k}(-)^{t} k_{(t)}(\beta-\gamma+k-2 t) \\
& \quad \times \frac{\beta^{[k-t]} \gamma^{[t]}(\psi-\beta)^{[t]}(\psi-\gamma)^{[k-t]}(\theta-\gamma-t)^{[n]}(\phi-\gamma-t)^{[n]}}{(\beta-\gamma-t)^{[k+1]} \psi^{[k]} \theta^{[n]} \phi^{[n]}} \\
& =\sum_{t=0}^{t=k}(-)^{t} k_{(t)}(\phi-\theta+k-2 t) \\
& \quad \times \frac{(\phi-\psi-k)^{[k-t]}(\theta-\psi-k)^{[t]}(\theta-\beta-k+t)^{[n]}(\phi-\beta-t)^{[n]}}{(\phi-\theta-t)^{[k+1]} \psi^{[k]} \theta^{[n-k+t]} \phi^{[n-t]}} \\
& =\sum_{t=0}^{t=k} k_{(t)} \frac{\beta^{[t]}(\theta-\psi-k)^{[t]}(\psi-\beta)^{[k-t]}(\theta-\beta)^{[n-k]}(\phi-\beta-t)^{[n]}}{\psi^{[k]} \theta^{[n-k+t]} \phi^{[n]}},
\end{aligned}
$$

these being all subject to the condition

$$
\theta+\phi=\beta+\gamma+k-n+1 \text {. }
$$

